Sociologie et sociétés

\title{
Le cyberespace, figure de l'utopie technologique réticulaire
}

\section{Pierre Musso}

Volume 32, numéro 2, automne 2000

Les promesses du cyberespace. Médiations, pratiques et pouvoirs à l'heure de la communication électronique

URI : https://id.erudit.org/iderudit/001521ar

DOI : https://doi.org/10.7202/001521ar

Aller au sommaire du numéro

\section{Éditeur(s)}

Les Presses de l'Université de Montréal

\section{ISSN}

0038-030X (imprimé)

1492-1375 (numérique)

Découvrir la revue

Citer cet article

Musso, P. (2000). Le cyberespace, figure de l'utopie technologique réticulaire. Sociologie et sociétés, 32(2), 31-56. https://doi.org/10.7202/001521ar d'utilisation que vous pouvez consulter en ligne. 


\section{$\triangle$ \\ Le cyberespace, figure de l'utopie technologique réticulaire}

\section{pierre musso}

CREDAP, Université Panthéon-Sorbonne - Paris I

12, Place du Panthéon

F-75231 Paris Cedex 05, France

Courriel: pierre.musso@datar.gouv.fr

«C'est à un combat sans corps qu'il faut te préparer, tel que tu puisses faire front en tout cas, combat abstrait qui, au contraire des autres, s'apprend par rêverie»

Henri M ichaux, Poteaux d'angle

A vec l e cyber espace (ou cyberspace), double contemporain d'Internet, triomphe A l'image d'un réseau universel connectant tous les individus à l'échelle planétaire et constituant une sorte de «cerveau planétaire», comme le nomme Joël de Rosnay, producteur d'une «intelligence collective», sel on la formule de Pierre Lévy. Ce que la science-fiction a imaginé semblerait se réal iser avec Internet, réseau de réseaux, porteur de toutes les mythologies contemporaines. La société elle-même serait désormais «société en réseaux», nous dit M anuel Castells.

La technique joue le rôle symbolique dans nos sociétés désenchantées. Ainsi le réseau est-il devenu un attracteur, contribuant à un réenchantement recherché dans les utopies technologiques. Le réseau, et son cortège de métaphores, est au centre de ces nouveaux dispositifs de la communication.

Avant tout, les termes employés, cyberespace et réseau, méritent d'être questionnés. De quoi parle-t-on avec le cyberespace? D'une «hallucination consensuelle», d'après la définition de son créateur, William Gibson? D'un objet immatériel non 
identifié? N ous essaierons de montrer qu'il s'agit d'un dispositif symbolique, miroir d'Internet, expression du «grand projet utopique universel » contemporain fondé sur la technologie, projet que Lucien Sfez distingue du «récit utopique» traditionnel (Sfez, 1995, p. 105-121). Quant au réseau, précisons que s'il peut être défini comme un lien, c'est-à-dire par sa fonction de liaison, voire de métaliaison, il demeure «lien delieux». II relie des pôles. II est l'expression d'une tension entre des pôles préalablement posés comme opposés. Dans le cyberespace, ces lieux reliés par les réseaux sont eux-mêmes des réseaux, à savoir des cerveaux-réseaux et des ordinateurs en réseau, formant «cerveau collectif». La construction du cyberespace repose sur trois présupposés : tout d'abord, le réseau est entendu comme interconnexion généralisée, ensuiteil y aurait des lieux, différents et se ressemblant, à savoir des cerveaux et des ordinateurs et, enfin, serait possiblel'hybridation entre homme et machine, du fait del'assimilation cerveauréseau-ordinateur.

Avec le cyberespace est mise en scène une symbolique de type religieux - une religion de l'immanence - fondée sur le réseau technique, notamment Internet. Plusieurs auteurs ont souligné cette dimension religieuse. Christian Huitema a bien titré son ouvrage Et Dieu créa Internet, et Kevin Kelly, rédacteur en chef adjoint de Wired, a pu décrire sa première visite à Internet comme une «expérience religieuse» (cité par Dery, 1997, p. 57).

Le cyberespace réactualise une mythologie du xix ${ }^{e}$ siècle, celle fondée par les saintsimoniens, liée au «désenchantement» de la religion chrétienne et à son déplacement sur l'industrie et les réseaux techniques. Le réenchantement de notre mode industriel s'est opéré depuis grâce aux technologies dont Internet semble aujourd'hui le paradigme.

C'est cet imaginaire du réseau dans la cyberculture que nous voudrions interroger dans cet article. On cherchera d'abord, à identifier quel ques structures de l'imaginaire du cyberespace mettant en jeu le réseau, puis on mettra en perspective cet imaginaire réticulaire du cyberespace, notamment à partir de la matrice philosophique saintsimonienne qui rend possible l'approche moderne du réseau.

On se propose d'analyser quelques «poncifs du cyberspace» (son idéologie en quelque sorte) en s'appuyant notamment sur quelques ouvrages de chercheursvulgarisateurs, notamment ceux de Pierre Lévy, Joël de Rosnay ou Nicholas N egroponte. Leur problématique reprend bien souvent les thématiques de la science-fiction, à commencer par William Gibson, ou celles de revues cultes nord-américaines, commeWired ou M ondo 2000 qui ont été à l'origine de cette littérature.

\section{i. le cyberespace, nouvel espace religieux}

Le cyberespace relie sans fin. II réescompte l'ordre du religieux (religare) et l'insère dans les technologies. II peut être défini comme un réseau de structures réticulaires mises en réseau entre elles. C'est un réseau au cube (au sens où Distler et Bressand avaient parlé de «Réseaupolis», réseau au carré). Il est l'espace d'inscription de la «matrice», du «cerveau planétaire» ou de «l'intelligence collective». Les structures qu'il 
met en réseau sont des cerveaux et des ordinateurs eux-mêmes «petits» réseaux (de neurones ou de puces électroniques) associés dans de plus vastes réseaux comme Internet et, in fine, interconnectés dans le «grand » réseau du cyberespace. Le cyberespace serait en quelque sorte, la limite de la pensée du réticulaire, dans sa version spirituelle.

Chez William Gibson, qui crée en 1983, le terme de «cyberspace» dans Neuromancien (référence au mot nécromancien, sorcier qui réveille les morts), tout est affaire de «neuroconnexion » (Gibson, 1988, p. 6). Le héros, Case, pirate en fuite, se connecte avec le cyberespace par une interface neurologique, en branchant son système nerveux sur «la matrice», une réalité virtuelle globale où les informations sont stockées sous forme d'illusions tangibles. La matriceest «l'éclatant treillis de logique qui se dévidait à travers un vide incolore» (ibid., p. 7). Case a vécu «pour l'exultation désincarnée du cyberespace» (ibid., p. 9), «branché sur une platine du cyberspacemaison qui projetait sa conscience désincarnée au sein de l'hallucination consensuelle qu'était la matrice» (ibid., p. 8). Autrement dit, Case a vécu l'expérience de la désincarnation «le corps c'était de la viande» - et il a pu quitter son corps pour traverser l'au-delà cyberspatial, guidé par le fantôme d'un pirate informatique mort synthétisé par ordinateur.

\section{a) Le cyberespace, espace réticulaire «spirituel»}

La première définition du cyberespace donnée par Gibson lui-même est la suivante: «Le cyberespace. Une hallucination consensuelle vécue quotidiennement en toute légalité par des dizaines de millions d'opérateurs, dans tous les pays, par des gosses auxquels on enseigne les concepts mathématiques... Une représentation graphique de données extraites des mémoires de tous les ordinateurs du système humain. Une complexité impensable. Des traits de lumière disposés dans le non-espace de l'esprit, des amas et des constellations de données. Comme les lumières de villes, dans le lointain » (ibid., p. 64).

L'hallucination imaginée par l'auteur de science-fiction est devenue progressivement un terme désignant le nouvel espace informationnel fait de réseaux ordinatiques et cervicaux mêlés. Chez Gibson, le cerveau et le systèmenerveux de Case sont connectés au réseau électronique, le cyberespace : le cerveau est extériorisé (en ordinateur cerveau artificiel), puis connecté. Émerge le thème du «crâne câblé» (wirehead) défini par le N ew Hacker's D ictionary, comme un pirate informatique spécialisé dans le détournement des systèmes de communication. Dans la Schismatrice, Bruce Sterling appelle aussi les M écanistes dotés de prothèses et connectés par ordinateur, des «wireheads» et dans les cercles cyberpunk, le terme est synonyme d' «aspirant cyborg»; le cyborg étant la connexion du cerveau individuel au cerveau planétaire artificiel (Dery, 1997, p. 356, note 179).

De son côté, le rédacteur en chef de la revueM ondo 2000 déclare: «Jepensequenous sommes engagés dans un processus d'info-connexion en vue de la construction d'un systèmenerveux global, d'un cerveau global »(citépar Dery, 1997, p. 58). Quant à Joël de 
Rosnay, il considère que «le cyberspace incarne le monde virtuel qui naît des informations échangées par les hommes dans les réseaux de communication. [... ] Le monde d'Internet est un cyberspace. II créé les conditions d'une nouvelle citoyenneté électronique... Mais lecyberspace est encore une jungle, bruissant demille dangers, où l'on peut se perdre. Un Far West numériqueau sein duquel pirates et escrocs évoluent à leur guise. Lecyberspace est un océan illimité, une terra incognita sur laquelleon s'aventure avec des cartes rudimentaires» (de Rosnay, 1995, p. 166-67). Le cyberespace est, tour à tour, une «incarnation» du mondevirtuel (dimension symbolique, voire religieuse), un nouveau territoire sans carte, une jungle où l'on seperd et s'aventure, et un nouvel espace public, citoyen ou marchand. Le cyberespace est un être hybride, à la fois une technique (Internet), une forme de la nature (jungle, territoire), une nouvelle forme sociale $d^{\prime}$ " «espace public» (pour la citoyenneté) et symbolique. Le cyberespace confond, en un seul mot-valise, technique, symbolique, nature et société. Qu'est-ce qui fait l'unité de ces significations? Si ce n'est l'idée «d'interconnexion » référée aux réseaux de communication, commel'indique la définition qu'en donne Joël de Rosnay dans son glossaire : «le cyberspace est un espace temps électronique créé par les réseaux de communication et les interconnexions entre ordinateurs multimédias» (de Rosnay, 1995, p. 334).

Espace de réseaux interréliés à l'infini, tel est le cyberespace. Pierre Lévy confirme cette réduction du cyberespace d'abord au «réseau », puis à la vague idée d'interconnexion : «Le cyberspace (qu'on appellera aussi le «réseau ») est le nouveau milieu de communication qui émerge del'interconnexion mondiale des ordinateurs [... ] L'une des idées, ou peut-être, devrait-on dire, l'une des pulsions les plus fortes à l'origine du cyberspace est celle de l'interconnexion. [... ] l'interconnexion tisse un universel par contact» (Lévy, 1997, p. 17 et p. 150-151). L'auteur précise mêmecette définition du cyberespace, entendu «comme l'espace de communication ouvert par l'interconnexion mondiale des ordinateurs et des mémoires informatiques» (ibid., p. 107). La réduction s'achève finalement dans la perception d'une «sensation d'espace englobant». Cettesensation rappelleétrangement la «communion », c'est-à-dire la communication entendue dans son sens étymologique, c'est-à-dire religieux, comme partage ou miseen commun.

C'est la même idée d'interconnexion qui sert de pivot à la définition de «la société en réseaux» de Manuel Castells: «II s'agit en premier lieu de définir le concept de réseau, caractéristique majeure de la société à l'ère de l'information. Un réseau est un ensemble de nœuds interconnectés. Un nœud est un point d'intersection d'une courbe par elle-même. La réalité d'un nœud dépend du type de nœud auquel il appartient » (Castells, 1998, p. 526).

Le cyberespace, identifiéà un espace fait uniquement de réseaux, est caractérisé par I'interconnexion sans fin. En effet, ce territoire n'a pas de topographie, mais uniquement une topologie. Si le cyberespace fait allusion au territoire, il est en fait, un espace sans histoire, un espace non territorialisé : il n'est pas une «terre-histoire». N euf, il est donc susceptible de toutes les représentations. Dans le cyberespace, terre inconnue (terra incognita) faite de réseaux, les lieux interconnectés sont eux-mêmes des «petits réseaux», à savoir des cerveaux et des ordinateurs. 
Ainsi le cyberespace a-t-il l'immensevertu d'évacuer tout ce qui gêne ou qui résiste, à commencer par le territoire. Or, un réseau technique ne se définit que dans son rapport au territoire physique, comme le rappelle Gabriel Dupuy : «Le réseau n'est pas la conséquence detelle ou telle innovation technologique. C'est un principe d'aménagement qui met en rapport des possibilités techniques et la desserte d'un territoire» (Dupuy, 1985, p. 6). Gabriel Dupuy énonce les propriétés du réseau, notamment la connexité et la connectivité. La première «est un concept topologique qui permet de caractériser le réseau de relations entre sous-systèmes d'un réseau territorial » (ibid., p. 68). Emprunté à la théorie des graphes, ce concept permet de distinguer trois types de réseaux : non connexes, connexes, plus ou moins fortement connexes. Un réseau est connexe, nous dit Dupuy, s'il «solidarise»l'espace socio-économique, s'il «crée de la solidarité» (ibid., p. 70), à l'exemple du réseau routier : il est non connexe dansle cas contraire (exemple des voies navigables). Autrement dit, la connexité est à la fois une figure de la densité du réseau et une notion socio-économique de solidarité liée au territoire physique.

La connexité est au réseau ce quel'organisation est au système. En effet, plus un système est organisé, donc complexe, plus sa structure réseautique est connexe : «La propriété première des réseaux dans leurs rapports au système territorial, est la connexité. C'est d'abord par la connexitéque le réseau solidarise les éléments d'un système et peut constituer un territoire» (Dupuy, 1985, p. 127).

Or, dans le cyberespace, le territoire n'existe pas. Le territoire rugueux, résistant est effacé. Ne subsiste qu'un espace lisse, fluide, de circulation. II s'agit d'un espace de réseaux informationnels; il n'y a ni histoire, ni lieux, il n'y a que des liens: «Les réseaux sont des structures ouvertes, susceptibles de s'étendre à l'infini, intégrant des nœuds nouveaux en tant qu'ils sont capables de communiquer au sein du réseau » (Castells, 1998, p. 526-527).

Cet espace de réseaux se développant sans fin est hybride, mi-humain, mi-machinique : il relie hommes et machines indistinctement parceque les réseaux sont tantôt des organismes, tantôt des artefacts. Le réseau confond en un tout la technique et le biologique : il appelle ainsi son extension virale, sur un mode biologique. Le cyberespace est un être hybride, mais vivant! Pour le philosophe postmoderne M anuel de Landa, «passéun certain seuil de connectivité, la membrane dont les réseaux informatiques recouvrent la planète commenceà "prendre vie". Des logiciels indépendants vont bientôt constituer des communautés computationnelles encore plus complexes, où ils traiteront I'un avec l'autre, commanderont, se disputeront les ressources, se féconderont et enfanteront spontanément des programmes et cetera» (cité par Dery, 1997, p. 55).

Pierre Lévy peut donc affirmer : «Il en est du cyberspace comme de certains systèmes écologiques» (1997, p. 131) ; «c'est un ordinateur dont le centre est partout et la circonférence nulle part, un ordinateur hypertextuel, dispersé, vivant, pullulant, inachevé : le cyberspace lui-même» (ibid. ; p. 52). De même le «cybionte» de Joël de Rosnay est-il «un superorganisme hybride, biologique, mécanique et électronique, incluant les hommes, les machines, les réseaux, les sociétés» (de Rosnay, 1995, p. 334). 
Mais le cyberespace est d'abord dual, car il est aussi le «support» de son double abstrait qu'il incarne, à savoir l'intelligence collective : "Le cyberspace, dispositif communicationnel interactif et communautaire, se présentejustement commeun des instruments privilégiés de l'intelligence collective» (Lévy, 1997, p. 31). Le cyberespace n'existeque dans le dualisme qu'il instaure entre le support matériel (infrastructure) et «l'intelligence collective» (dimension spirituelle).

«En somme, l'extension du cyberspace accompagne et accélère une virtualisation générale de l'économie et de la société. Des substances et des objets, on remonte aux processus qui les produisent. Des territoires, on saute en amont, vers les réseaux mobiles qui les valorisent et les dessinent. Des processus et des réseaux, on passe aux compétences et aux scénarios qui les commandent, plus virtuels encore» (ibid., p. 58-59). Pierre Lévy décrit un système en étages au sommet duquel le virtuel est à son maximum : del'infrastructure au virtuel/spirituel, il refait le trajet del'ascèse religieuse vers l'esprit. Cet ordre du cyberespace était déjà décrit par le rapport N ora-M inc de 1978 sur L'informatisation de la société française, pour dessiner la «société d'information » : en bas les réseaux et en haut l'intelligence. L'ordre cyberspatial n'échappe pas à la traditionnelle métaphore corporelle qui place depuis l'Antiquité les fonctions intelligentes «en haut» et les autres, «en bas». Le rapport Nora-M inc livrait une première représentation de la société d'information ${ }^{1}$ qui a ordonné durablement les discours sur la communication depuis la fin des années 1970, en fixant l'impératif du passage à la «société d'information » : «La scène sociale traditionnelle tendra à se désarticuler, au fur et à mesure du passage de la société industrielle organique à la société d'information, polymorphe» (Nora et M inc, 1978, p. 114).

L'instauration du cyberespace comme espaceillimité des réseaux permet de circuler hors contraintes, dans l'espace pur, éthéré, «virtuel», des réseaux informationnels. Par un exercice d'exorcisme, tout devient possible dans cet espace idéel (idéal), une fois le territoire oublié.

Mais exit aussi lecorps, car seul lecerveau est sollicité dans l'aventure cyberspatiale.

\section{b) Le « réseau pensant »}

Dans le cyberespace, cerveaux et ordinateurs sont donc branchés entre eux, comme I'avait déjà rêvéWiener dans Cybernetics (1948), car ils relèvent tous deux d'une même théorie unitaire qui rend compte du fonctionnement des machines et des organismes. Ils sont à la fois décomposés en parties identifiables (les puces électroniques équivalent aux neurones) et «interconnectés» pour donner naissance à une petite total ité «intelligente» (le cerveau et l'ordinateur) et extensibleà une «grande totalité» construite par analogie, à savoir le «cerveau planétaire» (reliant cerveaux et ordinateurs intercon-

1. «Schématiquement, disent les rapporteurs, la vienationales'organisera sur trois étages, correspondant à trois fonctions, à trois systèmes de régulation et donc d'information. L'étage proprement régalien où se déterminera le projet collectif... L'étage où s'organiseront et se confronteront les projets relationnels et culturels: ce sera le domaine de "I'agora informationnelle". L'étage du marché, reposant sur le système de prix : celui où s'expriment et s'arbitrent les désirs spontanés des groupes... En réalité ces étages interféreront» (Nora et M inc, 1978, p. 124-125). 
nectés) dotée d'une «intelligence collective». Ce jeu d'analogie repose sur deux identifications préalables : le cerveau est un ordinateur et le cerveau dispose, comme lui, d'une structure neuronale réticulaire support de l'activité intellectuelle.

Le syllogisme fondateur du cyberespace est très simple:

- Proposition majeure : le cerveau fonctionne comme un ordinateur et réciproquement, l'ordinateur fonctionne (et «pense») comme un cerveau.

Tous deux relèvent d'une théorie unitaire ayant pour objet la connexion d'éléments en réseau : le cerveau est un réseau de neurones et l'ordinateur est composé de puces mises en réseau.

- Proposition mineure : avec Internet se développe un réseau de réseaux mondial par connexion des ordinateurs (qui en sont les composants)

- Conclusion : il est possible de connecter les cerveaux humains et les ordinateurs entre eux, grâce à des hyperréseaux reliés à l'échelle planétaire. Ainsi peuvent être obtenues une hybridation homme-machine et une «intelligence collective» dans et par le cyberespace.

Ces raisonnements s'appuient sur l'anal ogie supposée entre le fonctionnement du cerveau et la structure réseautique, analogie bien antérieure à l'invention de l'ordinateur et des technologies contemporaines de communication. En effet, dès les débuts de la médecine, le réseau est associé au corps et ce lien traversera toute l'histoire des représentations du réseau, désignant tantôt le corps dans sa totalité comme agencement de flux ou de tissus, tantôt une partie de celui-ci, notamment le cerveau. Galien (131-200 ap. J.-C.) parlait déjà de l'esprit ou du cerveau comme d'un merveilleux filet, «retemirabili ». La métaphore galiénique du cerveau-réseau s'est installée durablement, et sera réactivée de façon récurrente chez D escartes, Diderot, Saint-Simon, Spencer ou dans la première cybernétique et le connexionnisme contemporain.

Sous le cerveau, se cache le réseau, et réciproquement, avec le réseau se profile le cerveau. Depuis D escartes et Saint-Simon (voir M usso, 1998 et 1999), la structure réticulaire explique le fonctionnement du système complexe; c'est la définition même du concept de réseau. Derrière le réseau technique de communication, il y aura toujours le réseau idéal-cognitif à atteindre, derrière le cerveau, il y aura le fonctionnement logique en réseau. Réseau et cerveau convergent dans la production partagée «d'intelligence» : le premier sur le mode artificiel, voire artificieux (Sfez, 1993), et le second sur le mode naturel. Georges Canguilhem avait bien souligné cette réversibilité de l'image cerveau-ordinateur : "On peut au choix parler de l'ordinateur comme d'un cerveau ou du cerveau comme d'un ordinateur » (Canguilhem, 1993, p. 19).

$\mathrm{Si}$, dès la mythologie, Gilbert Durand constate une «étroite concomitance entre les gestes du corps, les centres nerveux et les représentations symboliques» (Durand, 1969, p. 51), c'est précisément cette image du «système nerveux» qui est devenue d'un usage ordinaire pour définir les techniques de (télé)communication depuis un siècle.

Comme l'a montré Armand M attelart (M attelart, 1994, p. 87-91), cette image du réseau-système nerveux, trouveune première formulation explicite chez H erbert Spencer (1820-1903), ancien ingénieur des chemins de fer devenu sociologue, dont l'organicisme 
identifie ordre biologique et ordre social. La théorie de Spencer emprunte l'opposition des saint-simoniens, notamment enfantinienne, entre «société organique» ou industrielle et «société militaire», et la pousse jusqu'au bout dans une vision organiciste du social. Spencer distinguait dans la société trois «appareils d'organes» : I'appareil producteur, le distributeur et le régulateur. Dansle corps humain, l'organe régulateur est le système nerveux qui diffuse de l'information dans l'organisme: dans la société l'équivalent du système nerveux est l'ensemble des moyens de communication, qu'il s'agisse de la poste, du télégraphe ou des agences de presse : «Le seul fil télégraphique, écrit Spencer, qui accompagne le système de chemin de fer dans toutes ses ramifications est le fil qui en arrêteou en excitele trafic, demêmequelenerf qui accompagne partout une artère est le nerf vaso-moteur qui y règle la circulation [... ]. Q uoique pour les fils télégraphiques en l'air on ait un autre moded'isolement, les fils souterrains sont isolés d'une manière qui a del'analogie avec celle qu'on observe dans les fibres nerveuses» (Spencer, 1890 , tome2, p. 82).

Dès 1896, cette métaphore système nerveux-réseau est reprise par R. Worms qui dans son ouvrage O rganisme et société, assimilait «les routes et les chemins de fer aux vaisseaux sanguins, le télégraphe aux nerfs, les machines aux muscles du corps social » (cité par Schlanger, 1970, p. 90).

Depuis le début du siècle, elle est utilisée de façon récurrente dans les télécommunications. Ainsi la trouve-t-on sous la plume de Theodor Vail, le patron d'American Telegraph and Telephone à ses origines, qui écrit dans le rapport d'activités de sa société de 1908, «lesystèmeBell s'est développé sous le signe d'un contrôle intelligent et d'une large structure jusqu'à s'intégrer au système nerveux de l'activité économique et de I'organisation sociale du pays (il est même devenu de fait ce système nerveux) » (Réseaux, 1992, p. 61).

Bien avant la cybernétique, Pierre Janet, professeur au Collège deFrance, écrivait dès 1923-1924, «le cerveau n'est qu'un ensemble de commutateurs» (Canguilhem, 1993, p. 15).

L'identification des réseaux de communication au système nerveux a été notamment réescomptée et vulgarisée par la première cybernétique qui rassemblait psychologues et mathématiciens, puis reprise lors de l'invention de l'ordinateur. Si depuis Galien, sous le cerveau, se cachait le réseau, désormais, derrière le réseau, se profile le cerveau : «Les motivations des premiers informaticiens sont liées au projet de construire une nouvelle machine qui serait un "cerveau artificiel" rapide et peut-être plus raisonnable quel'homme» (Breton, 1991, p. 67-69).

Cette identification des réseaux de télécommunications au système nerveux fut vulgarisée notamment par Warren M cCulloch et Walter Pitts dans leur célèbre article de 1943, «Un calcul logique des idées immanentes dans l'activité nerveuse» dans lequel ils affirment «Le systèmenerveux est un réseau deneurones» (cité par Pélissier et Tête, 1995, p. 62). Ce qui est essentiel c'est «le caractère en "tout ou rien" de l'activité nerveuse», car son fonctionnement peut être formalisé au moyen de la logique propositionnelle: «II semble que le comportement de chaque réseau peut être décrit en ces termes si on y ajoute des outils logiques plus compliqués pour les réseaux contenant des 
boucles; en outre pour toute expression logique satisfaisant certaines conditions, on peut trouver un réseau qui se comporte selon le modèle décrit» (ibid.).

Dans son article de 1949, «Du cerveau comme calculateur», M cCulloch va directement à l'identification du cerveau et de l'ordinateur : «Le cerveau peut être apparentéà un calculateur numérique consistant en dix milliards de relais appelés neurones», donc «le cerveau est une machine logique» (cité par Pélissier et Tête, 1995, p. 192-193). Or, le cerveau humain étant «de beaucoup le plus complexe des calculateurs» (ibid.), l'analyse de son mode de fonctionnement vaudra pour tout autresystème logique. Sachant que dans le cerveau «chaque relais est une cellule vivante» (ibid., p. 193), le référent des deux formes de «relais-cellule» est un réseau de télécommunication. Inversement, le cerveau devient un modèle pour l'ingénieur; même si «les ingénieurs ne pourront espérer rivaliser avec la nature [... ] Les concepteurs de machines à calculer seraient heureux d'échanger leurs meilleurs relais avec des cellules nerveuses» (ibid., p. 195). Lemodèle de M cCulloch et Pitts associeunemachine logique et une machine biologique; comme le souligne Lucien Sfez, «les représentations mentales, tout comme les comportements, ont le même processus logique, celui des réseaux et de leurs connexions neuronales» (Sfez, 1990, p. 81).

Wiener fixe le paradigme de la «ressemblance fondamentale entre le système nerveux et les machines électroniques» (Wiener, 1971, p. 90) ; David LeBreton souligne que «Wiener met en place, sous l'égide de l'information, un paradigme crucial de l'extrême contemporain. L'identité règne à ses yeux entre les hommes et les mécanismes autorégulés. Leurs qualités respectives sont secondaires au regard de l'information qu'ils traitent» (LeBreton, 1999, p. 180). Von Neumann et Turing poussent la comparaison à son terme.

A près avoir souligné que la principale intuition des premiers cognitivistes (H. Simon, N . Chomsky, M . M insky et M cCarthy) «était quel'intelligence (y compris I'intelligence humaine) est tellement proche de ce qu'est intrinsèquement un ordinateur que la cognition peut être définie par la computation » (Varela, 1989, p. 35-36), Francisco Varela considère que la cognition fonctionne «par l'entremise d'un réseau d'éléments inter-connectés capables de subir des changements structuraux au cours d'un historiquenon interrompu » (ibid., p. 112). Les réseaux (de réseaux) de neurones sont la structure explicative de la cognition.

Dès qu'on évoque la figure du réseau, la métaphore organistique originelle rôde, de façon toujours active; comme le rappelle Lucien Sfez, «sous les processus heuristiques en réseau, sous le traitement abstrait des programmes réside encore la figure du corps réel » (Sfez, 1990, p. 400).

Derrière le cerveau, il y a toujours le fonctionnement logique en réseau et derrière le réseau de communication, il y a le réseau idéal-cognitif à atteindre. L'un renvoie à I'autrejusqu'à fusionner dans la figure contemporaine du cyberespace et du «réseau intelligent». Désormais, le réseau de communication peut produire de «l'intelligence». Ainsi Alvin Toffler peut nous entretenir du «réseau conscient» : non seulement le réseau est «une sorte d'équivalent de système nerveux de notre société», mais surtout, après 
avoir été «inintelligent» à un «stade primitif», «le réseau commençait à avoir une certaine conscience delui-même» (Toffler, 1991, p. 144). Le réseau de communication est au corps social ce qu'est le réseau nerveux au corps humain ; son fonctionnement assure sa survie. M ais, à la différence des précédents, les nouveaux réseaux seraient «intelligents», comme l'affirme Toffler : «Les réseaux qu'établirent M orse, la Western Union, Bell et autres en tendant leurs filsétaient inintelligents, pour ne pas dire parfaitement stupides» (ibid., p. 143). Le réseau, défini comme «système nerveux», alimente les représentations dominantes et s'identifie à sa métaphore. Chez Joël de Rosnay, le «Cerveau planétai re» confond en un tout indistinct, réseaux télématiques et «réticulation sociale» (de Rosnay, 1986, p. 24). Désormais, le réseau Internet illustre ce «cerveau planétaire», et «Nous sommes en train d'assister - et de participer "del'intérieur" - à la construction du systèmenerveux et du cerveau planétaire du micro-organisme sociétal » (de Rosnay, 1995, p. 85 et 79). Les réseaux techniques sont des organismes vivants qui réalisent l'utopie d'une intelligence collective. À tel point qu'un ingénieur peut écrire très directement dans un ouvrage sur les télécommunications du $x x i^{\mathrm{e}}$ siècle que «Les réseaux sont des êtres vivants, disposant de nombreux organes fonctionnels (transmission, commutation, accès) mettant en œuvre des technologies qui ont connu et vont connaître de nombreuses mutations» (Feneyrol, 1996, p. 68). Triomphe du réseau-organisme!

\section{c) La sainte trinité réticulaire}

Trois types de réseaux techniques structurent l'imaginaire de la communication au $x x^{e}$ siècle : d'abord, le réseau de radiodiffusion dit «point à masse» (d'un point d'émission à une multitude de récepteurs), ensuite le réseau téléinformatique constitué de la connexion aléatoire d'ordinateurs, dit de «n vers $n$ » (d'un nombrequelconque d'émetteurs vers un nombre quelconque de récepteurs) et enfin, le réseau de téléphonie commuté, dit «point à point» (d'un émetteur/récepteur à un autre). Le premier type est le modèle pyramidal de la radiodiffusion et suggère la domination de l'émetteur sur une multitude de récepteurs. Lesecond modèle, celui del'informatique, suggère l'interconnexion anarchique des acteurs. Quant au troisième, le modèle du réseau téléphonique maillé et commuté, il évoque l'image égalitaire de tous les acteurs branchés. Cette typologie des réseaux de communication issue de la science des ingénieurs vise, lorsqu'elle est importée dans le champ social, la valorisation du réseau télé-informatique «interactif», «voire égalitaire», dont Internet est le modèle.

Avec l'instauration du cyberespace, est réescomptée cette «sainte trinité» des réseaux techniques qui valorise le réseau téléinformatique «den vers $n$ », voire de «tous vers tous», opposés aux médias traditionnels de radiodiffusion où domine l'émetteur et où les récepteurs sont censés être «passifs». Cette distinction «technique» dans la structure des réseaux véhicule toute une vision del'organisation sociale : à tel point que les sciences sociales l'ont empruntée à la science des ingénieurs², la transformant en un véritable poncif.

2. La distinction techniqueinterne à la «science desingénieurs» est ainsi présentée dans un texte cosigné par Lelu, M arcovici et Kretz (1983, p. 35) ingénieurs des télécommunications, et intitulé «Penser les réseaux» : sont décrites les trois formes principales du réseau, c'est-à-dire les « trois pôles qui balisent le champ des 
Ainsi Pierre Lévy affirme-t-il que

Le cyberspace encourage un échange réciproque et communautaire alors que les médias classiques mettent en œuvre une communication unidirectionnelle où les récepteurs sont isolés les uns des autres. II existe donc une sorte d'antinomie, ou d'opposition de principe, entre les médias et la cyberculture.

\section{Il insiste:}

Une des principales significations de l'émergence du cyberspace est le développement d'une alternative aux médias de masse. J'appelle médias de masse les dispositifs de communication qui diffusent une information organisée et programmée à partir d'un centre, en direction d'un grand nombre de récepteurs anonymes, passifs et isolés les uns des autres. Presse, cinéma, radio et télévision classiques sont les représentants typiques de tels médias. Or le cyberspace ne met pas en jeu des centres diffusant vers des récepteurs, mais des espaces communs où chacun peut apporter son lot et puiser cequi l'intéresse, des sortes de marchés del'information où les gens se rencontrent et où l'initiative appartient au demandeur (Lévy, 1997, p. 249 et 294) $)^{3}$.

Quant à Nicholas N egroponte, il affirme aussi dans L'homme numérique :

La télévision est l'exempletype d'un média dans lequel toutel'intelligence se trouveau point d'origine. Le diffuseur décide, et le spectateur se contente de prendre ce qu'on lui donne. En fait, au centimètre cube, votre poste de télévision est peut-être bien l'appareil le plus bête que vous ayez chez vous. [... ] Dans un journal, l'intelligence toute entière se trouve également à la source. [... ] Est-ce bien inéluctable? II faudrait créer des ordinateurs [... ] qui liraient les journaux et regarderaient la télévision pour nous et ne retiendraient que ce qui nous intéresse. Ce type d'intelligence peut se situer à deux endroits différents. Elle peut se situer chez l'émetteur ou bien votre système d'édition d'information se situe chez le récepteur. [... ] Dans ce cas, l'intelligence se situe à l'extrémité réception, et l'émetteur passif envoie sans discrimination tous les bits à tout le monde. L'avenir n'est pas à l'un ou à l'autre, mais aux deux (Negroponte, 1995, p. 33-35).

Pour sa part, Joël de Rosnay différencie le «modèle mécanique inspiré de la physique» qui «conduit à une organisation pyramidale et à un mode de commandement hiérarchique», du «modèle biologique, plus récent » et qui «favorise une organisation en réseau » et du «modèle chaotique qui est adapté à la complexité de son environnement» (de Rosnay, 1995, p. 226). À son tour, Jean-Louis Le M oigne (1989)

systèmes de communication électronique, et tout système réel constitue un composé de ces trois pôles à des dosages divers $[\ldots]$ :

- lepôle "tout vient du centre": ce sont les systèmes de diffusion par réseaux hertziens ou câblés, suivant leprincipe "tout le monde est arrosé en mêmetemps"; aucune remontée d'information n'est organisée sur support électronique vers les systèmes centraux (émetteurs ou têtes de réseau);

- le pôle "tout local": c'est ce qu'on appelle la "privatique", c'est-à-dire les dispositifs individuels de stockage d'information et detraitement de celle-ci [... ];

- le pôle "réseau commuté" contrastant avec les deux pôles précédents qui incarnent des dissymétries complètes entre le centre et la périphérie, il existeuneforme de réseau respectant unesymétrie complète entre les usagers, à la fois émetteurs et récepteurs, dont l'archétype est constitué par le réseau téléphonique commuté. »

3. Pierre Lévy décrit aussi «trois grandes catégories de dispositifs communicationnels : un-tous, un-un et tous-tous. La presse, la radio, la télévision sont structurées par le principe un-tous: un centre émetteur envoie ses messages à un grand nombre de récepteurs passifs et dispersés. La poste ou le téléphone organise 
à la recherche de «la mémoire des réseaux», repère aussi trois modèles technico-organisationnels de réseaux : le «réseau anarchique», le «réseau hiérarchique» et le «réseau intelligent $»^{4}$.

Tous ces schémas ternaires de réseaux techniques, organisationnels ou sociaux puisés dans la science des ingénieurs vont ordonner la plupart des représentations sociotechniques de l'imaginaire des réseaux. Dès 1948, la sociologie des organisations importait ces trois schémas issus de la science des ingénieurs. Alex Bavelas publie en 1948, A M athematical M odel for Group Structures (cité par Ardoino, 1993), où il établit trois figures du réseau simulant autant de structures et d'organisations différentes : la pyramide ou réseau centralisé, le réseau maillé et le réseau en chaîne. Ces trois figures du réseau technique de communication et des organisations hantent désormaisl'imaginaire de la communication. Le cyberespace récupère cette «saintetrinité» pour valoriser la figure du réseau téléinformatique detype Internet, comme modèle de la connexion libre et égalitaire.

A contrario, la trinité réticulaire appliquée au social a l'immense avantage de disqualifier les structures pyramidales et hiérarchiques. Exit I'Institution, et notamment l'État, grâce au réseau interactif et au cyberespace.

\section{d) La communion cyberspatiale et le retour des anges}

Le cyberespace est un puissant dissolvant symbolique (une «hallucination consensuelle») qui réunit les partisans de la liberté du marché et de la société civile dans I'anti-étatisme, les libéraux et les libertaires, réalisant ainsi l'égal ité de tous les membres du cyberespace et des internautes branchés, à égal ité avec les grands groupes, unis dans la fraternité des communautés virtuelles. Le cyberespace dissout le territoire, le corps, mais aussi la mémoire et l'État. Exit le politique. Tout ce qui résiste est éliminé.

Grâce au réseau, la démocratie sera électronique et «le politique disparaîtra» peut annoncer Jacques Attali (Attali et Castells, 1998), la société sera faite de réseaux, pour reprendrela vision de $M$ anuel Castells qui considère que «l'un des aspects majeurs de

des relations réciproques entre interlocuteurs, mais seulement selon des contacts d'individu à individu ou de point à point. Le cyberspace met en œuvre un dispositif communicationnel original puisqu'il permet à des communautés de constituer progressivement et de manière coopérative un contexte commun (dispositif tous-tous) » (1977, p. 75).

4. «Soit une organisation de $\mathrm{N}$ acteurs se proposant d'établir un réseau de communication entre eux afin que, par leurs échanges d'information, elle soit en mesure d'assurer une tâche (un calcul par exemple) donnée. Deux réseaux extrêmes viennent ai sément à l'esprit du concepteur: le réseau $A$ (anarchique) assure que chacun des $\mathrm{N}$ acteurs est reliéà chacun des $\mathrm{N}$ autres (il peut communiquer avec lui-même). La matrice du graphe représentant ce réseau est "pleine": le réseau compte $N 2$ interrelations. [... ]

Le réseau $\mathrm{H}$ (hiérarchique) assure qu'un acteur, présumé doté d'une grande capacité de computation, est relié aux $\mathrm{N}-1$ autres qui lui transmettent toutes les informations en leur possession (seule une colonne de la matrice du grapheest pleine), $\mathrm{N}-1$ interrelations [... ] cetteorganisation est beaucoup plus fragile [... ]. La prise en compte de la fonction mémorisation associable à tout réseau conduit à élaborer unenouvelle forme du réseau, la forme $M$, et par là une forme nouvelle d'organisation [... ] on peut concevoir un réseau $M$ centré mémoire dont l'économie globale sera peu différente de celle d'un réseau de type $H$, et dont la robustesse sera del'ordre de celle d'un réseau detype A [... ] architecture de réseau intelligent, intelligent parce que mémorisant» (Le M oigne, 1989, p. 180-182). 
la société informationnelle est la logique du réseau » (Castells, 1998, p. 43 et note). Les réseaux constituent, selon l'auteur, «la nouvelle morphologie sociale de nos sociétés» (ibid., p. 525) et le réseau fonctionne comme structure explicative du système capitaliste contemporain. «Les réseaux convergent vers un métaréseau de capitaux» (ibid., p. 531), le métaréseau est la nouvelle figure du pouvoir. L'enjeu de ces images dominantes du réseau et autre «métaréseau » est d'évacuer le politique et sa forme étatico-nationale, en valorisant la circulation généralisée des flux. M anuel Castells peut aussi affirmer que «les réseaux détruisent le contrôle étatique sur la société et sur l'économie. Ce qui est fini, dans l'étape actuelle, c'est l'État souverain, national » (Attali et Castells, 1998).

Cette vision anti-étatique de type libéralo-libertaire croise l'idéologie des internautes que Christian Huitéma résume en ces termes, exploitant toujours la «sainte trinité» technologique des réseaux de communication :

L'Internet n'est pas, commela radio ou la télévision, un média à sens unique. Ce qu'il y a de plus révolutionnaire dans le réseau, c'est précisément la possibilité pour chacun d'être à la fois consommateur et une source d'information. [... ] Loin d'être une institution de contrôle, I'Internet sera au contraire un instrument de liberté, permettant à l'homme moderne de secouer le joug des bureaucraties. Quand l'information passe les frontières entre pays, les États ont beaucoup plus de peine à gouverner par le mensonge et la propagande. On voit déjà, dans les entreprises informatisées, la communication s'affranchir des voies hiérarchiques et, peu à peu, les hiérarchies s'aplatir, la déférence craintive et la certitude arrogante céder la place au dialogue égalitaire (Huitema, 1995, p. 180-183).

Le réseau, «par essence» anti-hiérarchique, devient synonyme d'auto-organisation et d'égalité, représentation dominante des internautes que porte leur revue culte Wired. L'internaute est censé mener un combat pour la liberté contre tous les organes de régulation, contre les opérateurs dominants (M icrosoft ou lef bi, par exemple) pour l'égalité, contre toutes les hiérarchies, à commencer par celles des États, et pour la fraternitémondiale des «communautés virtuelles». Liberté, égalité et fraternité : I'utopie sociale de 89 (1789/1989) se réaliserait enfin grâce à l'utopie technique réticulaire. «Le cyberspace peut apparaître comme une sorte de matérialisation technique des idéaux modernes» écrit Pierre Lévy (1997, p. 302).

Soit la version philosophique de cette utopie technologique du réseau planétaire que M ichel Serres (1994a) ne nomme même plus utopie, mais «Pantopie» dans La légende des anges. Grâce aux réseaux de communication, Michel Serres voit émerger une «Pantopie» universelle qui remplacerait l'utopie, et par laquelle tous les lieux sont en chaque lieu et chaque lieu en tous lieux. À tel point que les «réseaux nous enchantent, mais comme des drogues» (Serres, 1994b, p. 16). Ainsi Michel Serres conclut-il sa Légende des anges par ce dialogue avec son lecteur :

Au lieu de tisser des réseaux de choses ou d'êtres, dessinons donc des entrelacs de chemins. Les Anges ne cessent de tracer les cartes de notre nouvel univers. [... ] Visibles et invisibles, construisant les messages et les messageries, esprits et corps, spirituels et physiques, des deux sexes et sans sexe, naturels et techniques, collectifs et sociaux, en désordre et en ordre, producteurs de bruit, de musique, de langue, intermédiaires, échangeurs, intelligence 
répandue dans les choses du monde et les artefacts... avouez que ces Anges sont insaisissables (Serres, 1994a, p. 293 et 296).

Êtres intermédiaires, à la fois matériels et spirituels comme l'avait déjà indiqué M ichel Chevalier dans la célèbre série d'articles de 1832 parus dans le journal Le Globe et intitulés Le système de la M éditerranée (M usso, 1999b), les réseaux deviennent les anges médiateurs du futur. Le réseau planétaire et le cyberespace sont les nouveaux anges de l'utopie technicienne qui relie les hommes à leur futur.

Le réseau, liaison technique, est une réserve sans fond de métaphores pour annoncer la venue de mondes nouveaux. Tel est aussi le point de vue développé par Joël de Rosnay : «Par l'intermédiaire des réseaux mondiaux interconnectés, privés, publics, commerciaux, militaires, réseaux de réseaux, ou réseaux locaux, se tissent irréversiblement les mailles d'une nouvelle forme de cerveau collectif. Cerveau hybride, biologique et électronique, et bientôt biotique» (de Rosnay, 1995, p. 166).

Cette thématique du «réseau pensant » ou du «cerveau planétaire» et del'universalisme est issue de Teilhard de Chardin (1881-1955) qui parlait de «conscience planétaire». Joël de Rosnay - comme Pierre Lévy et toute la cyberculture - reprend à son compte la notion de «noosphère», désignant «la sphère naissante de l'esprit humain, après la biosphère», et la définit comme «l'ensemble des productions dématérialisées». Chez Teilhard,

à l'intérieur de la noosphère en voie de compression, une nouvelle chaîne se dessinerait, particulièrement centrale et directe, la cérébralisation se renfermant sur elle même dans un processus de self-achèvement, une autocérébralisation de l'humanitédevenant l'expression la plus concentrée du rebondissement réfléchi de l'évolution (Teilhard de Chardin, 1956, p. 56).

Le «point oméga » est, selon Teilhard, la concentration ultime de l'esprit dématérialisé :

L'hommeest apparu selon le même mécanisme (géographiqueet morphologique) quetoute autre espèce. (Effet sélectif d'absorption par le temps des portions les plus fragiles.) Cependant dès l'origine, nous saisissons certaines particularités qui dénotent en lui une vitalité supérieure... la céphalisation ou cérébralisation (ibid., p. 86).

La pensée de Teilhard a repris du service avec les idéologies N ew Age et postmoderne. Les leaders de la pensée New Age, notamment M arilyn Ferguson, auteur du best-seller Les enfants du Verseau, revendiquent la paternité du célèbre jésuite paléontologue français dont les conceptions sur la noosphère, enveloppe spirituelle de la Terre, ou la naissance d'un cerveau collectif, influencent directement les théoriciens du cyberespace. Le culte du réseau par les évangélistes du New Age est très proche dans sa description de celle du cyberespace : le réseau, dit M arilyn Ferguson, est

I'antidote de l'aliénation. II engendre suffisamment de pouvoir pour refaire la société. II offre à l'individu un soutien affectif, intellectuel, spirituel et économique. C'est un lieu d'accueil invisible, un moyen puissant de modifier le cours des institutions, en particulier le gouvernement. Le réseau ressemble à un psychisme collectif articulé au tissu social. La conspiration du Verseau est un réseau de nombreux réseaux dont la vocation est la transformation sociale (Ferguson, 1981, p. 163). 
Quant au postmodernisme de Lyotard, Lucien Sfez a bien montré sa complicité avec le projet noosphérien de Teilhard, pour

former des êtres hybrides qui seront chargés de poursuivre une tâche : celle de survivre à la mort des mondes [... ] ou bien s'enroulant sur soi, devenir une sphère de pen sée suiréférentielle, un monde pensant, se mouvant éternellement dans l'univers. nous ne sommes pas loin de la surhumanité et des cyborgs en tous genres. (Sfez, 1995, p. 335-337).

\section{e) La dissolution du corps}

Ultime étape de l'ascèse cyberspatiale : le complément du branchement du cerveau-ordinateur sur la matrice, c'est la désincarnation du corps. Dans les réseaux du cyberespace, le statut du corps se modifie : l'esprit domine (comme cerveau-réseau-ordinateur), il s'immerge, navigue et disparaît «dans» les mailles du filet. Ce sont des «anges» désincarnés qui communiquent : «Lorsqu'on communique par ordinateur interposé, on communique comme les anges», dit Stewart Brand : alors «communiquent des intelligences désincarnées, supérieurement intimes» (cité par Dery, 1997, p. 67-68). Ainsi est restauréle dualisme qui survalorise l'esprit-cerveau, au détriment du «corps-viande». II s'agit de devenir des «purs esprits», vieux rêve mystique réalisé par l'ordinateur en réseau.

Le rédacteur en chef de la revue culte Wired dit du N et et des réseaux que ce sont des «eco-systèmes nerveux, des choses qui nous connectent à un au-delà - littéralement physiquement - au-delà de nos corps». Rappelons que chez Gibson, «La cléde la psychologie de Case, c'est son aliénation du corps, de la viande» (Gibson, 1988, p. 260), car «Pour Case qui n'avait vécu que pour l'exaltation désincarnée du cyberspace, ce fut la Chute. Dans les bars qu'il fréquentait du temps de sa gloire, l'attitude élitiste exigeait un certain mépris pour la chair. Le corps, c'était de la viande. Case était tombé dans la prison de sa propre chair » (Gibson, 1988, p. 9).

Si lecerveau est survalorisé, branché sur l'ordinateur, «la matrice» ou le «cerveau collectif», alors le corps est dévalorisé par cette ascèse vers l'hypercérébralisation de «l'ultra-humain » de Teilhard. Le cyberespace, comme le dit S. Bukatman, est «célébration de l'esprit. [... ] C'est un royaume où le mental est libéré des limites corporelles, un lieu favorableà la toute puissance de la pensée» (cité par Le Breton, 1999, p. 139). Exit le corps superflu, désincarné, dissout même, dans le cyberespace. C'est cette «humanité sans corps» que critique finement l'anthropologue David LeBreton : «Les pesanteurs du corps sont effacées. [... ] Les internautes sont sur un plan d'égalité du fait justement de la mise entre parenthèses du corps. Le cyberspace est l'apothéose de la société du spectacle, d'un monde réduit au regard, à la mobilité de l'imaginaire, mais à l'arraisonnement des corps devenus inutiles et encombrants. »Cette mise en suspension ou entre parenthèses ouvre à d'autres usages du corps : «Le cyberspace représente un monde où le temps, affranchi de la durée, se convertit en espace d'informations pures ne requérant plus la corporéité humaine» (ibid., p. 140 et 142). Dans le cyberespace s'incarne un cogito pur.

En même temps que l'ordinateur est anthropomorphisé, le corps est digitalisé. Yves Stourdzé avait bien annoncé cela dès 1979, dans son livre intitulé Les ruines du 
futur, quand il soulignait la puissance des puces et non des fortifications, des réseaux et non des polarités. «Ce qui s'avance au-delà même de l'industrialisation, c'est l'irrésistible digitalisation des corps», c'est-à-dire «l'extermination corporelle».

Avec le cyberespace, exit le territoire, le corps ou l'institution : tout ce qui est encombrant, résistant ou rugueux est finalement numérisé et dissout dans la symbolique communicationnelle. Le cyberespace est un puissant dissolvant de tout ce qui gêne I'ascèse et la cérébralisation. Espace spirituel des réseaux immatériels, il est le support numérique d'unevision religieusefaited'un syncrétisme postmoderne, mêlant l'idéologie New Age et la philosophie de Teilhard de Chardin. II permet aux technologues de théoriser (et de rêver) leurs pratiques du réseau. En invitant à l'ascèse, il réescompte toutes les figures intermédiaires mi-homme, mi-machine, les cyborgs et autres cybiontes ou, plus prosaïquement, les nouvelles formes de l'angélisme. Et ce, d'autant plus aisé ment que la figure du Réseau assure traditionnellement une fonction de passage entre des entités (homme/machine) ou des mondes (réalité/cyberespace) posés comme opposés.

A près avoir décrit quel ques aspects du cyberespace et de sa figure réticulaire, nous voudrions interpréter cet imaginaire en commençant par sa mise en perspective historique. Cette nouvelle mythologie postmoderne et New Age est en fait une vieille histoire. Parce que le mythe fonctionneà la récurrence, le cyberespace ne fait que réinvestir et réescompter, à l'heure d'Internet, une vieille utopie fabriquéeil y a deux siècles, lorsqu'il fut déjà question de réagir au «désenchantement» religieux du monde.

\section{ii. décryptage de l'imaginaire du réseau cyberspatial}

En effet, au moment du «désenchantement du monde» et de la vidange de la religion, sont inventés les grands réseaux techniques modernes (télégraphie, chemins de fer) en tant qu'ils modifient le rapport à l'espace et au temps. Ces réseaux émergent à la charnière des $x$ v $\mathrm{ii}^{\mathrm{i}}{ }^{\mathrm{e}}$ et $\mathrm{xix} \mathrm{e}^{\mathrm{e}}$ siècles commela réponse prométhéenne qui fait advenir le monde moderne, libéré de sa référence supranaturelle. Ces réseaux de transports, de l'énergie et de la communication nous émerveillent à leur tour : de «la Fée électricité» aux « réseaux intelligents», ces techniques réenchantent quotidiennement notremonde.

Le concept moderne de réseau est forgé à l'occasion du grand chambardement provoqué par la naissance d'une nouvelle épistéméà la fin du xv viii e siècle. Dans ce contexte, le corps est libéré de sa référence à Dieu, il s'autonomise, devient modèle de rationalité, mais aussitôt il est réinséré, voire capturé, dans l'institution hospitalière et administrative. II sort de sa soumission à Dieu pour entrer dans celle de l'institution, qu'il s'agisse de l'État ou de l'Usine.

La grande rupture qui fait advenir un nouveau concept de réseau, c'est sa «sortie» du corps. Alors que, depuis la médecine hippocratique et galiénique, le réseau était associé au corps, il ne va plus être seulement observé sur ou dans le corps humain, il pourra désormais être construit par l'ingénieur. Dans Les lieux dans l'homme, Hippocrate pensait l'ordre des flux invisibles de la circulation des humeurs comme une structure réticulaire cachée, expliquant les manifestations externes du corps. Celien 
invisible est à la fois interne et externe au corps humain, car il renvoie à l'ordre caché du Cosmos dans lequel tout corps s'inscrit. Dès les débuts de la médecine, le réseau fut lié au corps, et ce lien traversa toute l'histoire des représentations du réseau, désignant tantôt lecorps dans sa totalité comme agencement de flux ou de tissus, tantôt une partie de celui-ci, notamment le cerveau (voir Musso, 1999b et 1999c).

M ais avec la Naissance de la clinique vers 1800 , le réseau est distingué du corps naturel, il devient un artefact, une technique autonome, indépendante du corps. Denaturel, le réseau devient artificiel. De donné, il devient construit. D'outil, il devient machine. L'ingénieur le conçoit et le construit, alors que le médecin l'observait. Le médecin qui considérait le corps comme un réseau, passe le relais à l'ingénieur qui fabrique des réseaux et les pense comme des organismes.

C'est à ce moment-là que la figure du réseau prend sa portée symbolique moderne, évacuant son rapport traditionnel au corps physique pour devenir un artefact produit par l'ingénieur prométhéen. Le mythe cyberspatial réescompte à son insu la mémoire de cette rupture entre le réseau et le corps intervenue à la fin du xvii $i{ }^{e}$ siècle pour aider au réenchantement technologique de notre monde industriel (voir Dagognet, 1995). Parmi les acteurs del'invention de ces mythes du monde technico-industriel, les saintsimoniens jouèrent un rôle décisif.

\section{a) La fondation saint-simonienne d'une utopie sociale réticulaire}

C'est Claude-Henri deSaint-Simon (1760-1825) qui produit la théorie de la nouvellevision «biopolitique» du réseau en opposant deux systèmes sociaux identifiés l'un au réseau de surveillance, le système «féodalo-militai re», et l'autre à la circulation généralisée des flux dans la société, le «système industriel ». La question du passage d'un système social à l'autrel'oblige à penser la transition et à produire pour cela le concept moderne de réseau.

Saint-Simon décèle deux catégories à l'origine de toute contradiction constitutive d'un phénomènequelconque : les «corps bruts» et «les corps organisés» qu'il oppose. Le corps brut est comme un «crible», c'est-à-dire un réseau qui laisse «filer» les fluides afin de retenir les solides; le corps organisé est un réseau qui assure la circulation des fluides. Corps brut et corps organisé sont comme les deux figures d'un réseau qui tantôt capte les solides, quand il est plongé dans un liquide (tel un filet), tantôt canalise les flux quand il est inscrit sur le sol (tel un réseau hydraulique). Ces deux faces de Janus du réseau renvoient sur le plan théorique au jeu de domination d'un des deux termes dans toute contradiction. II suffit donc d'un «petit déplacement» dans leur équilibre pour passer d'une domination à l'autre. Ainsi le corps organisé se solidifie et meurt quand la circulation des fluides cesse; le corps brut se fluidifie sous l'action de la chaleur. La totalitéconcrète et rationnelle d'un corps résulte du déséquilibre entre fluides et solides.

Si le cristal est une première forme de réseau, conformément à la théorie cristallographique fondée par l'abbé-académicien René-Just H aüy (1743-1827), la circulation

du fluide en définit une autre variété, et leur relation est une troisième variante de réseau, intermédiaire entre les deux états. 
Le réseau peut être tour à tour cristal, organisme et être hybride : une chose, la chose posée comme opposée et leur relation. Autrement dit, selon Saint-Simon, le réseau peut recouvrir des formes variées, à la fois solide, fluide et état intermédiaire entre les deux. Étonnante plasticité de la figure du réseau qui peut revêtir des formes diverses : un état, son inverse et le passage d'un état à l'autre.

Grâceà cettelogique du réseau, Saint-Simon dispose d'un outil d'analysetrès puissant pour élaborer une «science politique» et fonder une nouvelle religion. Dans le champ politique, cette logique permettant de penser toute forme de passage est transformée en théorie de la transition sociale. Comment, s'interroge Saint-Simon, assurer le passage paisible et insensi ble du système présent au système futur? I s'agit, tout simplement, de favoriser la circulation del'argent dans la société. Établir l'immédiation de la circulation du «sang-argent» revient à organiser le corps social comme un corps humain. Saint-Simon définit la circulation dans le réseau - sanguin ou étatique comme la condition de la vie ou de la bonne administration, c'est-à-dire comme la condition du changement social. La transition au système industriel espéré se résume à libérer la circulation del'argent dans le corps de l'État. La même institution, à savoir l'appareil d'État, peut être tantôt un lieu d'exercice de la force - tel est le «système féodal »-, tantôt un lieu de circulation del'argent, et voilà le «système industriel ». Un simple déplacement opéréà l'intérieur del'État entraîne du même coup le passage du système féodal-bureaucratique au système industriel-démocratique, «du gouvernement des hommes à l'administration des choses», selon la célèbre formule d'Enfantin, vulgarisée par le marxisme.

Dès 1769 , dans Le rêve de d'Alembert ${ }^{5}$, Diderot avait fait émerger une figure du réseau qui symbolisel'ambivalence du contrôle et de la circulation. La symbolique du réseau était bâtie depuis la mythologie, sur l'ambivalence de la continuité et de la rupture, mais avec la vision biopolitique des Lumières, le réseau est pris dans une nouvelle ambivalence et désigneles deux grandes figures du contrôle social : la surveillance centralisée du panopticon que critique M ichel Foucault dans Surveiller et punir, et la circulation permanente qui fonctionne au «contrôle continu » qu'analysera Gilles Deleuze (1990, p. 236). Symbole de circulation et de continuité, le réseau renvoie immédiatement à son contraire, la panne, l'arrêt, la crise, la saturation, le bouchon, le court-circuit, et finalement la mort. La symbolique du réseau fait basculer de la surveillance à la circulation ou l'inverse.

Pour Saint-Simon, l'établissement du système industriel devient œuvre divine, symbole du «N ouveau Christianisme», titre de son dernier ouvrage rédigé en 1825 et demeuréinachevé. L'industrialisme saint-simonien del'atelier ou del'usine se ressource à la morale chrétienne paulinienne de l'amour du prochain. Dans ce «Nouveau Christianisme», la communion se réalise dans le travail des associés appliqué à la

5. Dans ce «rêve», Mademoiselle de l'Espinasse compare le corps humain à une toile d'araignée et le docteur Bordeu, que Diderot met en scène, assimilele corps à «un réseau qui se forme, s'accroît, s'étend, jette unemultitude de fils imperceptibles» et il ajoute «les fils sont partout, il n'y a pas un point à la surface de votre corps auquel ils n'aboutissent». Selon qu'on setrouve au centre, c'est la mémoire et le despotismequi triom- 
planète entière, pour la fécondation de la nature avec les réseaux de communication. Par ce travail des frères associés, la planète peut être reconfigurée comme un organisme idéal composé de réseaux artificiels la métamorphosant. La pratique religieuse saintsimonienne consiste à tracer sur le corps de la France et de la planète, c'est-à-dire sur son territoire, des réseaux pour assurer la circulation de tous les flux dans la société.

Le réseau symbolise définitivement - en acte et en représentation - lelien scellé entre les trois éléments de la religion saint-simonienne, l'association, la communication et la communion. La cyberculture et les évangélistes contemporains d'Internet et du cyberespace ne font que réactiver cette symbolique moderne (et non «postmoderne»), à partir de leur fétichisme du réseau. Le culte du réseau technique fut activement élaboré par les disciples de Saint-Simon dans les années 1830-1860 en Europe. Alors, le réseau trouve dans le discours saint-simonien son mythe modernel'identifiant à une révolution sociale et démocratique.

\section{b) L'utopie technologique réticulaire des saint-simoniens}

Les saint-simoniens s'emparent de la philosophie bâtie par leur maître pour penser et organiser à l'échelle sociale un culte religieux des réseaux. Les disciples mettent en scène la communion dans leur Église, «l'association des frères», dans leurs ateliers et séminaires de travail, et la communication dans leur politique des réseaux.

Le concept de réseau devient le symbole de l'association universelle. M ichel Chevalier (1806-1879) systématise cette conception des réseaux dans le célèbre articlemanifeste, «Le système de la M éditerranée» paru dans Le G lobe du 12 février 1832. II y écrit :

Aux yeux des hommes qui ont la foi quel'humanité marche vers l'association universelle, et qui se vouent à l'y conduire, les chemins de fer apparaissent sous un tout autre jour. [... ] Dans l'ordre matériel le chemin de fer est le symbole le plus parfait de l'association universelle. Les chemins de fer changeront les conditions de l'existence humaine.

Le passage de la domination des hommes à l'association des frères ne pourra se réal iser qu'avec le développement des réseaux de communication par la communion de I'O rient et de l'Occident. Le réseau permet de «passer » et de «dépasser » la lutte de I'Orient et de I'O ccident. II unit les deux, la chair et l'esprit, la femme et l'homme. La communion Orient-Occident est de mêmenaturequecelle dela chair et del'esprit dans la religion chrétienne. L'opérateur symbolique de cettefusion, sorte d'Eucharistie, est le réseau qui joue dans le «N ouveau Christianisme» un rôle équivalent à celui du Christ dans la religion traditionnelle, un lieu de transsubstantiation entre l'esprit et le corps.

La religion saint-simonienne s'affirme comme celle de la communion par les réseaux, qu'ils soient «spirituels», comme les réseaux bancaires du crédit, ou «matériels», comme les voies de communication. M ichel Chevalier précise que ces

phent, al ors qu'à la périphérie, règnent la communication et l'anarchie. Il suffit dès lors, de se déplacer du centre à la périphérie, telle l'araignée sur sa toile, pour «passer » de la mémoire à la communication et du despotisme à l'anarchie. Car, vu du centre, le réseau est surveillance et, vu de la périphérie, il devient communication. 
réseaux ont été jusqu'ici une affaire d'ingénieurs alors que leur portée politique est décisive, car ils contribuent à «l'association universelle». Le réseau est conçu à la fois comme une technique qui fait lien et comme un opérateur politico-moral qui fait sens. Le réseau est bien plus qu'une technique et un instrument de passage, il devient I'outil symbolique et pratique de la religion saint-simonienne. Les réseaux sont des symboles : convoquer le réseau comme objet revient simultanément àl'effacer comme technique, pour dévoiler sa vérité comme lien social, en acte dans «l'association universelle».

À la différence de Saint-Simon, Michel Chevalier transforme donc le réseau en objet-symbole: le réseau technique produit par lui-même du changement social. Chevalier ira même jusqu'à écrire les phrases fondatrices del'idéologie moderne de la communication :

Améliorer la communication, c'est travailler à la libertéréelle, positive et pratique. [... ] c'est faire de l'égalité et de la démocratie. Des moyens de transport perfectionnés ont pour effet de réduire les distances non seulement d'un point à un autre, mais égal ement d'une classe à une autre (Chevalier, 1836, vol. ii , p. 3).

Le réseau technique permet la communication, la communion et la démocratisation par la circulation égalitaire des hommes. La réduction géographique des distances physiques, voire l'interchangeabilité des lieux, grâce aux voies de communication, vaut réduction des distances sociales, c'est-à-dire démocratie. Le développement des réseaux est déjà identifiéà une «révolution politique».

Lors du passage de l'exposé de la doctrine saint-simonienne à la définition d'un culte du réseau, les disciples ont fait le chemin inverse de leur maître : ils partent du concept de réseau à l'achèvement duquel Saint-Simon était parvenu. Cette inversion de cheminement a eu pour effet de transformer une «semie utopie» sociale, selon la formule de Raymond Ruyer (Ruyer, 1950, p. 213), en utopie technologique pleine et entière. Ainsi Enfantin peut-il écrire: «Nous avons enlacé le globe de nos réseaux de chemin de fer, d'or, d'argent, d'électricité! Répandez, propagez, par ces nouvelles voies dont vous êtes en partie les créateurs et les maîtres, l'esprit de Dieu, l'éducation du genre humain » (cité par Pinet, 1898, p. 165-166). Cette déclaration résume le sens del'action des saint-simoniens : les réseaux de communication ont été réalisés en tant quepratique religieuse pour «enlacer le globe». Les réseaux sont des médiateurs de l'esprit divin. Leur développement est un véritable acte d'amour de la Terre fécondée par la technique, un enrichissement de la nature par le réseau. L'utopie sociale devient utopie technicienne. Depuis la reformulation saint-simonienne, l'utopie technologique ne cesse de se réaliser en une vulgate universelle du réseau, réinvestie de façon récurrente avec chaque innovation technique (chemin defer, électricité, téléphone, informatique, télématique), jusqu'à Internet.

Cristallisant un rapport, les saint-simoniens introduisent un fétichisme du réseau. $C^{\prime}$ est cette vision qui perdure dans les discours de la religion communicationnelle contemporaine : le réseau technique, notamment I'hyper- réseau Internet (réseau de réseaux planétaire), vaut désormais démocratie, circulation, égalité... 
Dans le «Nouveau Christianisme» industriel, le réseau joue un rôle équivalent à celui du Christ dans la religion traditionnelle, un lieu de transsubstantiation entre l'esprit et le corps, comme dans le cyberespace. M acLuhan avait justement souligné cette fonction christique des technologies de communication, indiquant que la convergence liée aux médias électroniques

pourrait produire cette universalité de la conscience annoncée par Dante lorsqu'il déclarait que les hommes neseraient que des fragments tant qu'ils nes'uniraient pas en une conscience globale. D'un point de vue chrétien, ce n'est qu'une nouvelle interprétation du corps mystique du Christ; et le Christ après tout, est le prolongement ultime de l'homme (cité par Dery, 1997, p. 57).

\section{c) La fonction symbolique du réseau cyberspatial}

Au-delà des variations de la figure réticulaire liée aux artefacts techniques censés la cristalliser, demeurel'invariant du réseau, c'est-à-dire la fétichisation du passage et de la transition, y compris sous sa forme christique. Le symbolique du réticulaire puise toujours à un fond mythologique récurrent, indiquant le Destin. Le réseau indique le passage vers le futur en mettant en évidence la continuité du fil du temps.

Le rite du passage, c'est bien sûr celui de Charon, marquant l'ambivalence de la vie (circulation des flux, le réseau fonctionne) et de la mort (panne, le réseau ne fonctionne pas) qui est consubstantielleà cettemétaphore et à la notion de «réseau ». La symbolique du réseau est d'abord structurée par le binômevie/mort, binôme dans lequel le réseau est tantôt identifiéà la panne, l'asphyxie ou la mort, tantôt assimiléà la vie. Selon le mode de fonctionnement du réseau, on est d'un côté ou de l'autre, la métaphore du réseau est d'abord bicéphale (surveillance dela circulation et circulation de la surveillance) et la figure du réseau est toujours prête à s'inverser (de la circulation à la surveillance, ou de la surveillanceà la circulation). Le réseau permet d'opposer uneforme généraleà la pyramide ou à l'arbre, linéaireet hiérarchisé, mais empêche de basculer dans lechaos et le désordre.

Dansl'ordre del'imaginaire, le réseau est l'intermédiaire : son être est letransitoire, sa substance est le passage. Sac à métaphores, il agit sur trois fronts à la fois, là est toute sa force : il se présente comme passage entre deux figures opposées qu'il instaure. II se déploie d'abord sur les deux versants du binôme réversible vie-mort ou quadrillagecirculation, puis assure le passage de l'un à l'autre des termes de ces couples de signes opposés. Le réseau peut être indifféremment un des deux termes d'uneopposition symbolique et le moyen terme «rationnel » liant les deux. II sépare puis relie lestermes séparés.

Puisant à la mythologie fondatrice du réseau entendu étymologiquement comme filet et tissu, le réseau, passage cristallisé, indique toujours le D estin et le sens, non plus celui de la verticalité de la flèche de la cathédrale tendue vers le supra-naturel, mais celui de l'interconnexion et de la liaison se développant sans limite, en «réseaux de réseaux» et «métaréseaux», en cyberespace.

Le réseau technique est comparable à une cathédrale dont la flèche indiquerait non plus l'au-delà, mais le futur terrestre promis ${ }^{6}$. Le réseau pointe l'avenir ici-bas,

6. Les premiers télégraphes aériens des frères Chappe étaient souvent installés sur la flèche des cathé 
celui de la «société en réseaux» dans les mailles de laquelle nous sommes déjà pris : il est devenu une sorte de temple de la religion communicationnelle. Là où le temple reliait ciel et terre, cette nouvelle cathédrale relie le présent et le futur, en promettant paix et démocratie par la circulation général isée de l'information.

Bien sûr, la symbolique du réseau demeure biface. Le Paradis peut se retourner en son contraire, l'Enfer du Contrôle(Big Brother) contre le Paradis de la Circulation, c'està-dire l'égalité des «frères internautes branchés», libéralo-libertaires qui ne cessent de célébrer l'auto-régulation et l'auto-organisation du réseau technique, identifiéà l'état na turel del'organismeplanétaire. Le réseau est devenu le nouveau médiateur vers le paradis terrestre d'une sociétéindustrielle ou informationnelle. II pointel'avenir ici-bas, et indique «l'entrée dans la société d'information et decommunication »; ainsi le programme d'action gouvernementale français dans le domaine des nouvelles technologies, lancé au début de 1998, viset-il à «préparer l'entrée de la France dans la sociétéd'information ».

Le réseau-cathédrale n'est pas ordonné par la verticalité, mais par une horizontalité toute terrestre et par la continuité des flux qu'il organise. De même que la cathédrale est incarnation du mystère, le réseau est présence du futur : il fait passer, en définissant notre place comme un passage. Désormais, les réseaux d'information, le cyberespace et Internet incarnent le passage, voire la vitesse du passage (cf. la «dromologie» évoquée par Paul Virilio).

La vérité de la société actuelle, c'est-à-dire la structure cachée de sa complexité est le réseau : tantôt il est «système nerveux», tantôt encore «cerveau planétaire» de la société, voire du monde. Comme le répète $M$ anuel Castells, «Cette base matérielle, constituée en réseaux, marque au fer rouge les processus sociaux dominants et façonne ainsi la structure sociale elle-même» (Castells, 1998, p. 527).

Le réseau est un passeur qui nous transmute en «passants», toujours plongés dans des flux (d'informations, d'images, de sons, de données... ). Le mouvement est continu : de même que la République platonicienne mettait chacun à sa place, la démocratie réticulai re met chacun dansune situation de passage, en le «branchant » à un réseau.

Lieu de passage permanent, le réseau permet à la fois d'indiquer que le futur est dans «la société de communication en réseaux» avec son double cyberspatial, mais que leprésent saturé de réseaux oblige à passer. Le présent est passage, transition, mouvement. Plus besoin d'opérer le changement social, il se fait en permanence. Puisque tout est réseau, puisque la société a sa vérité dans les réseaux, alors on est constamment dans le passage social. La société de réseaux est une société de la circulation généralisée. Inutile de penser de nouvelles utopies sociales, l'utopie technologique se charge de réaliser le changement social. Ainsi, I'utopie technologique décharge-t-elle I'utopie sociale de son fardeau. Le cyberespace, fruit de la science-fiction, devient un projet utopique universel réalisable; car l'utopie technologique est réaliste, comme le montre bien Lucien Sfez (Sfez, 1995, chapitre 3).

drales ou des églises: sortes de points hauts laïcisés. La Révolution n'annonçait-elle pas que «la machine télégraphique va prendrela placedu clocher de la cathédrale» (cité par Flichy, 1991, p. 22). 
Ainsi le réseau est-il devenu la fin et le moyen pour penser et réaliser la transformation sociale, voire les révolutions de notre temps. L'imaginaire du réseau est une façon de faire l'économie des utopies de la transformation sociale, d'opérer un transfert au sens psychanalytique. Alors que Saint-Simon forgea ce concept pour penser le changement social, il est devenu un moyen de ne plus y penser. Tel est le propre de la fétichisation des concepts. C'est pourquoi le réseau de communication porte une si épaisse mémoire symbolique, toujours disponible pour être réactivée avec chaque rêverie ou innovation technique.

Pour conclure, plaçons-nous avec Pierre Legendre, du point de vue du «déterminisme symbolique» (Legendre, 1999, p. 71), car ce déterminismelà a l'immense avantage de prendre le contrepied du déterminisme technologique dominant.

Le réseau est messager du Futur ou du Destin. En effet, le réseau a toujours eu partie liée avec le tissu et, commel'a notéGilbert Durand, «les instruments et les produits du tissage et du filage sont universellement symboliques du devenir » (Durand, 1969, p. 369). Le réseau désigne ce «vide symbolique» qui est censé le fonder : cette place vide est celle du Futur ou du Destin.

Tout comme la cathédrale est le symbole de ésus et la célébration de son corpsune «poétique de l'incarnation», dit Georges Duby qui précise: «Dans le corps du Christ, Dieu et homme, l'univers corporel et l'univers spirituel reviennent à leur unité native. Jésus - et la cathédrale qui en est le symbole - sont ainsi désignés comme le centre dont tout procède, où tout s'éclaire» (Duby, 1976, p. 220 et 175) - lecyberespace incarnela «M atrice», en désincarnant le corps. Cequ'il dissout d'un côté (leterritoire, le corps ou l'institution), il le réincarned'un autre côté (la matrice, le cyborg et autres «êtres hybrides»).

Le cyberespace, espace del'intermédiaire, des êtres hybrides et del'angélisme technicien, est le messager de la «matrice» ou du «cerveau planétaire», qui définissent la place vacante de Dieu ou de l'État. Legendre aime à citer cette expression de Dante visant à nommer le «principe qui manque» (Legendre, 1999, p. 17). Parce que «toute société doit prendre en charge une représentation du pourquoi ?» (ibid., p. 26), le symbolique a besoin de la représentation pour apparaître, il a besoin d'un messager : le prêtre, l'ange, le Christ ou l'État, c'est selon la Référence à convoquer.

Comment se réalise aujourd'hui, dans les sociétés ultra-modernes, (postindustrialistes, postmodernes, New Age, etc.) à l'heure d'Internet et du cyberespace, la theêatralisation du Videsymbolique, la mise en scène du pourquoi, si longtemps géré par la religion et ses prêtres, puis ensuite par l'État et les mécanismes de la représentation politique?

Le nouveau M essager, le nouvel Hermès de nos sociétés contemporaines, se réalise par et dans les techniques de communication, en tant qu'elles sont du symbolique pétrifié et industrialisé, et notamment dans les réseaux dont Internet et le cyberespace sont les actuelles figures.

La «M atrice», «l'intelligence collective» et le «cerveau planétaire» occupent la place du «principe qui manque» pour tenter de réenchanter notre monde industriel. 
SOCIOLOGIE ET SOCIÉTÉS VOL. XXXII.2

Le cyberespace est bien une «hallucination consensuelle», commel'a défini Gibson, qui théâtralise dans la technique le vide symbolique. II désincarne le monde réel pour mieux incarner la M atrice, nouvelle divinité de l'ère hypertechnicienne. î

\section{résumé}

Cet article interroge l'imaginaire du réseau dans la cyberculture. Avec le cyberespace, double contemporain d'Internet, triomphe l'image d'un réseau universel connectant tous les individus à l'échelle planétaire. Ce que la science fiction a imaginé, semblerait se réaliser avec Internet, porteur de toutes les mythologies contemporaines. Le cyberespace met en scène une symbolique de type religieux fondée sur le réseau technique. Parmi les acteurs majeurs de l'invention de ce mythe moderne, les saint-simoniens jouèrent un rôle décisif. Le cyberespace théâtralise dans la technique, le vide symbolique. Il désincarne le monde réel pour mieux incarner «la Matrice», nouvelle divinité de l'ère hypertechnicienne.

\section{summary}

This paper examines the imaginary of the networks in cyberculture. In cyberespace, contemporary double for the Internet, the image of a universal network connecting all individuals on a global scale triumphs. What was imagined by science fiction seems to have found its realisation in the Internet, which is the carrier for all contemporary mythologies. Cyberespace is the stage for a religious type of symbolism based on technical networks. Among the major actors in the invention of this modern myth, Saint-Simonians have played a decisive role. Cyberespace is dramatised in technique, a symbolic vacuum. It disembodies the real world in order to better incarnate "the Matrix", the new divinity of the hyper-technical age.

\section{resumen}

Este artículo interroga el imaginario de la cibercultura. Con el ciberespacio, doble contemporáneo de Internet, triunfa la imágen de una red universal que conecta a todos los individuos a escala del planeta. Lo que la ficción científica ha imaginado parecería realizarce con Internet, portador de todas la mitologías contemporáneas. El ciberespacio pone en escena una simbólica de tipo religioso fundada en la red técnica. Entre los actores mayores de este mito moderno, los sansimonianos jugaron un rol decisivo. El ciberespacio teatraliza en la técnica el vacío simbólico. Él desencarna el mundo real para encarnar mejor "la Matriz", nueva divinidad de la era hipertécnica.

\section{bibliographie}

At tal i, Jacques et M anuel Cast el s (1998), dialogue dans le supplément «Multimédia» du quotidien Libération du 12 juin.

A r d o ino, J. (1993), art. «Réseaux, structures et canaux de communication », in Lucien Sf ez (dir.), Dictionnaire critique dela communication, Paris, pu France, vol. 2, p. 614-617.

Br essand, Albert et Catherine Dist I er (1995), La planèterelationnelle. Essai, Paris, Flammarion.

Bressand, Albert et Catherine Dist I er (1986), Le prochain monde. Réseaupolis, Paris, Seuil, «Odyssée». Br et on, Philippe (1991), «L'informatique», in Lucien Sf ez (dir.), La communication, Paris, Cité des sciences, puf. 
Cangu il h em, Georges (1993), «Le cerveau et la pensée», in Georges Canguilhem, historien des sciences, Paris, Albin M ichel, coll. «Bibliothèque du Collège international de philosophie».

Cast el I s, M anuel (1998), L'ère del'information, t. I : La sociétéen réseaux, Paris, Fayard.

Ch eval ier, Michel (1836), Lettres sur l'Amérique du Nord, Paris, Gosselin, 2 volumes.

Dagon et , François (1995), L'invention du monde. L'industrie : pourquoi et comment?, Fougères LaVersanne, Encre Marine.

Del euze, Gilles (1990), Pourparlers, Paris, Éditions de Minuit.

De Rosnay, Joël (1995), L'homme symbiotique. Regards sur letroisièmemillénaire, Paris, Seuil.

De Rosnay, Joël (1986), Le cerveau planétaire, Paris, Olivier Orban.

Der y, M ark (1997), Vitesse virtuelle. La cyberculture aujourd'hui, Paris, Éditions Abbeville.

Dider ot, Denis (1769), Le rêveded'Alembert, in Euvresphilosophiques, (éd. : Paul Vernière) ; Paris, Éditions Bordas, coll. «Classiques Garnier», 1990.

Du by, Georges (1976), Le temps des cathédrales. L'art et la société980-1420, Paris, Gallimard, coll. «Bibliothèque des histoires».

D u puy, Gabriel (1985), Systèmes, réseaux et territoires. Principes de réseautique territoriale, Paris, Presses de l'École nationale des ponts et chaussées.

Dur and, Gilbert (1969), Les structures anthropologiques de l'imaginaire, 10e édition, Paris, Dunod.

Fen ey r ol , Michel (1996), Les télécommunications : réalités et virtualités. Un avenir pour lexx ie siècle, Paris, Masson, coll. «cnet - enst ».

Fer guson, Marilyn (1981), Les enfants du Verseau. Pour un nouveau paradigme, Paris, Calmann-Lévy.

Fl ich y, Patrice (1991), U ne histoire de la communication moderne. Espace public et vie privée, Paris, Éditions La Découverte, coll. «H istoire des sciences».

Gibson, William (1988), Neuromancien (trad. de l'américain par Jean Bonnefoy), Paris, J'ai Lu, coll. «sf ». H uit ema, Christian (1995), Et Dieu créa Internet, Paris, Éditions Eyrolles.

Le Br et on, David (1999), L'adieu au corps, Paris, Éditions M étailié.

Legendr e, Pierre (1999), Sur la question dogmatique en O ccident, Paris, Fayard.

Le M oigne, Jean-Louis (1989), «La mémoire du réseau : tout s'écoule... et pourtant», in Information, culture et société: la montée des réseaux, actes du colloque international, Grenoble, Université des sciences sociales de Grenoble, Réseau t ns Éditeur, 9-12 mai.

Lévy, Pierre (1997), La cyberculture. Rapport au Conseil del'Europe, Paris, Éditions O dile Jacob.

Lévy, Pierre (1994), L'intelligence collective. Pour une anthropologie du cyberespace, Paris, La Découverte.

Lel u,A., J.C. M ar c ovici et F. Kr et z (1983), «Penser les réseaux», in P. Flichy et G. Pineau (dir.), Images pour le câble. Programmes et réseaux de vidéocommunication, Paris, c n et -ina/La Documentation française.

$M$ at t el art , Armand (1994), L'invention dela communication, Paris, Éditions La Découverte.

M usso, Pierre (1999a), Saint-Simon et le saint-simonisme, Paris, puf , coll. «Que sais-je?».

M usso, Pierre (1999b), «La symbolique du réseau », Q uaderni, nº 38.

M u sso, Pierre (1999c), «La généalogie de la notion de réseau », Art Press, numéro spécial «Internet all over».

M u sso, Pierre (1998), Télécommunications et philosophiedes réseaux. La postérité paradoxale de Saint-Simon, $2^{e}$ édition, Paris, puf .

N egr o pont e, Nicholas (1995), L'hommenumérique, Paris, Robert Laffont.

N or a, Simon et Alain M inc (1978), L'informatisation dela société: rapport à M . lePrésident de la République, Paris, Le Seuil.

Pel issier , Aline et Alain Têt e (1995), Sciences cognitives (1943-1950), Paris, puf , coll. «Psychologie et sciences de la pensée»

Pin et, Gaston (1898), Écrivains et penseurs polytechniciens, Paris, Paul Ollendorff Éditeur.

Revue Réseaux, (1992), n 56, I ssy-Les M oulineaux, novembre-décembre.

Ruyer , Raymond (1950), L'utopie et lesutopies, Paris, puf .

Sch I ang er , Judith-E. (1970), Les métaphores de l'organisme, Paris, Librairie philosophique Jean Vrin.

Ser res, M ichel (1994a), La légende des anges, Paris, Flammarion.

Ser res, M ichel (1994b), Atlas, Paris, Julliard. 
Sf ez, Lucien (1995), La santé parfaite. Critiqued'une nouvelle utopie, Paris, Seuil.

Sf ez, Lucien (1993), art. «Artificieuse intelligence», in Dictionnaire critiquedela communication, tome1, Paris. Sf ez, Lucien (1990), Critique dela communication, $2^{e}$ édition, Paris, Seuil.

Spencer , H erbert (1883-1890), Principes de sociologie, 2 tomes, Paris, Germer, Baillière \& Co.

St o u r d ze, Yves (1999), Les ruines du futur, Paris, Sens \& Tonka.

Teil hard de Ch ar din, Pierre (1970), Le phénomène humain, Paris, Seuil.

Teil hard de Char din, Pierre (1956), La place del'homme dansla nature, Paris, Albin Michel.

Tof fl er , Alvin (1991), Les nouveaux pouvoirs? Savoir, richesse et violence à la veille du xxie siècle, Paris, Fayard.

Var el a, Francisco J. (1989), Connaître. Les sciences cognitives. Tendances et perspectives, Paris, Seuil.

W iener , N orbert (1971), Cybernétique et société, Paris, u gé, coll. «10/18». 